Ombretta Turriziani ＯRCID iD: 0000-0001-6400-9168

\title{
Seroprevalence of group B Coxsackieviruses: retrospective study in an Italian population
}

Ilaria Sciandra ${ }^{\mathrm{a}}$, Francesca Falasca ${ }^{\mathrm{b}}$, Paola Maida ${ }^{\mathrm{c}}$, Giulia Tranquilli ${ }^{\mathrm{b}}$, Daniele Di

Carlo $^{\mathrm{b}}$, Laura Mazzuti ${ }^{\mathrm{b}}$, Taulant Melengu ${ }^{\mathrm{b}}$, Gianluigi Giannelli ${ }^{\mathrm{a}}$, Guido Antonelli ${ }^{\mathrm{b}}$,

Ombretta Turriziani ${ }^{\text {b,* }}$

${ }^{a}$ National Institute of Gastroenterology “S. de Bellis”, Research Hospital, Castellana Grotte, Italy

${ }^{\mathrm{b}}$ Virology Unit, Department of Molecular Medicine, Sapienza University, Rome, Italy

${ }^{c}$ Infectious Diseases Unit, Department of Public Health and Infectious Disease, Sapienza University, Rome, Italy

Correspondence: ombretta.turriziani@uniroma1.it

Department of Molecular Medicine, “Sapienza” University, Rome, Italy

Viale dell’Università 31, 00185 Rome, Italy

Phone +39 06 49974298; Fax +39 0649974296

Running title: Group B Coxsackieviruses Seroprevalence

This article has been accepted for publication and undergone full peer review but has not been through the copyediting, typesetting, pagination and proofreading process, which may lead to differences between this version and the Version of Record. Please cite this article as doi: 10.1002/jmv.26096.

This article is protected by copyright. All rights reserved. 


\begin{abstract}
Purpose. Group B Coxsackieviruses (CVB) include 6 serotypes (B1-6) responsible for a wide range of clinical diseases. Since no recent seroepidemiologic data are available in Italy, the study aim was to investigate CVB seroprevalence in a wide Italian population.
\end{abstract}

Methods. The study retrospectively included 2,459 subjects referring to a large academic hospital in Rome (Italy) in the period 2004-2016. Seroprevalence rates and neutralizing antibodies (nAb) titers were evaluated in relation to years of observation and subjects' characteristics.

Results. Positivity for at least one serotype was detected in $69.1 \%$ of individuals. Overall, the prevalent serotype was B4, followed by B3 (33.3\%), B5 (26.2\%), B1 (12.7\%), B2 (11.0\%), and B6 (1.7\%). For B2, a significant decrease in seroprevalence over years was observed. Positivity to at least one virus was $25.2 \%$ in children aged 0 2 years, but significantly increased in pre-school (3-5 yr) (50.3\%) and school (6-10 yr) children (70.4\%). Higher nAb responses for B3 and B4 were observed in children aged 3-5 years.

Conclusion. A high overall CVB prevalence was found. Type-specific variations in prevalence over time probably reflect the fluctuations in circulation typical of Enteroviruses. Children are at greater risk for CVB infection given the high number of seronegative subjects aged 0-10 years.

Keywords. Coxsackievirus, seroprevalence, neutralizing antibodies, epidemiology, Enterovirus.

This article is protected by copyright. All rights reserved. 


\section{Introduction}

Group B Coxsackieviruses (CVB) are members of the family Picornaviridae, genus Enterovirus, species Enterovirus B, including 6 serotypes (B1-6). The acute infection, generally asymptomatic, is responsible for a wide range of diseases in children as well as in adults. Clinical presentations can be mild (flu-like or febrile symptoms, hand, foot and mouth disease ${ }^{1}$ ) or severe (meningitis ${ }^{2}$, acute flaccid paralysis ${ }^{3}$, pancreatitis ${ }^{4}$, acute myocarditis and pericarditis ${ }^{5,6}$ ). CVB seem also able to establish persistent infection in heart ${ }^{7}$ and pancreatic islets ${ }^{8}$, and the reactivation could be linked to disease development ${ }^{6}$. Interestingly, CVB infection has been proposed as immunologic trigger for the development of insulin-dependent diabetes ${ }^{9,10}$, although currently there are no confirmed causal evidences. In a recent study, however, a vaccine for B1 serotype conferred protection against virus-induced type 1 diabetes in a mouse model ${ }^{11}$.

CVB diffusion is ubiquitous and B1-5 serotypes display different circulation over the years $^{12-15}$. Changes in circulating types may develop as large-scale outbreaks, as observed in 2007-2009 with a B1 epidemic that caused fatal cases of neonatal myocarditis in the USA and Korea ${ }^{16,17}$. Essentially, CVB reflect the epidemic potential of Enteroviruses, pointing out the importance of surveillance.

Despite the widespread diffusion and the serious clinical presentations of CVB, seroepidemiologic data are limited worldwide. The last Italian work on this topic was published nearly 30 years ago ${ }^{18}$. To fill this gap, a retrospective analysis on CVB seroprevalence was performed on 2,459 individuals over a 13-year-long period. Neutralizing antibodies (nAb) titers were evaluated for all six serotypes, giving an 
insight into local CVB prevalence trends and investigating the immune status of different age-groups.

\section{Materials and Methods}

Patients and specimens. A total of 2,459 subjects hospitalized or visited at Umberto I University Hospital (Rome, Italy) from 2004 to 2016 were retrospectively included in the study. Information about age was available for 2,114 individuals. Patients' sera were routinely analyzed for suspected CVB infection following a physician's request. Clinical information was available for 486 patients, who showed one or more of the following symptoms: chest pain, cardiopathy, endocarditis, pericarditis, myocarditis, pericardial effusion, encephalitis, meningitis, pancreatitis, rash, fever, lymphadenopathy, arthralgia, gastroenteritis, or pneumonia.

Procedures performed in the study were in accordance with the ethical standards of the institutional and national research committee and with the 1964 Helsinki declaration and its later amendments or comparable ethical standards. For this type of retrospective study, formal consent is not required.

Micro-neutralization assay. Samples were screened with a micro-neutralization assay to detect neutralizing antibodies (nAb) against all six CVB. The serotypes were provided by the Virology Unit at Cisanello University Hospital (Pisa, Italy). After inactivation at $56^{\circ} \mathrm{C}$ for 30 minutes, sera were twofold serially diluted from 1:8 to 1:512 and tested in duplicate in a 96-well plate. Fifty $\mu$ l of B1-B6 virus with 100 TCID $_{50}$ were mixed with $50 \mu 1$ of each serum dilution and incubated at $37^{\circ} \mathrm{C}$ for 1 hour in $5 \% \mathrm{CO}_{2}$. To each well $100 \mu \mathrm{l}$ of $\mathrm{KB}$ cell suspension (human epithelial carcinoma) $\left(3 \cdot 10^{5}\right.$ cells $\left./ \mathrm{ml}\right)$ were added and the plate was incubated at $37^{\circ} \mathrm{C}$ in $5 \%$ $\mathrm{CO}_{2}$. Cell control and virus back titration were included, and a titration was performed 
to determine the virus titer. The presence of cytopathogenicity was evaluated after 4 days. The nAb titer was expressed as the geometric mean of the highest serum dilutions able to inhibit the viral cytopathic effect. Titers greater than or equal to 1:32 were considered positive. For titers higher than 1:512, the test was repeated adding further serum dilutions.

For 319 patients, a second serum sample was collected and sent to the laboratory after 10-15 days. Both the first and the second sample were then screened by microneutralization assay. The nAb geometric mean of the titers obtained for the 2 samples was used for the analysis. Patients with a 4-fold increase of nAb titers in the second sample were diagnosed with acute CVB infection and were not included in the study.

Statistical analysis. Seroprevalence and 95\% confidence intervals (CI) according to Wilson method were calculated. Chi-squared and Mann-Whitney tests were used to analyze the differences in seroprevalence and nAb titers between groups, respectively. The trends of seroprevalence over time were analyzed using the chi-squared test for trend. Statistical tests were conducted two-sided at a significance level of 0.05 using GraphPad Prism version 7.00 for Windows (GraphPad Software, La Jolla California USA).

\section{Results}

Subjects' characteristics. This study included 2,459 individuals (males: 54.7\%, 1,346/2,459) referring to a large academic hospital in Rome from 2004 to 2016. The 2,114 subjects with available information on age were divided into 11 age-groups. Age ranged from 1 month after birth to 97 years (mean age: 32.3 \pm 25.2 years).

Coxsackievirus B1-6 seroprevalence. Positivity for at least one serotype was found in $69.1 \%$ of individuals (95\% CI:67.2-70.9). Considering the whole 13-years-long This article is protected by copyright. All rights reserved. 
period of study, the prevalent serotype was B4 (45.2\%, 95\% CI: 43.3-47.2), followed by B3 (33.4\%, 95\% CI: 31.5-35.2) and B5 (26.2\%, 95\% CI: 24.5-27.9). Lower seroprevalence was observed respectively for B1 (12.7\%, 95\% CI: 11.4-14.1), B2 (11.0\%, 95\% CI: 9.8-12.3) and B6 (1.7\%, 95\% CI: 1.3-2.3) (Figure 1).

Significantly higher nAb levels were found for B3 and B4 compared to all other viruses $(p<0.0001)$, for B5 respect to B1 and B6 $(p<0.0001)$, and for B2 compared to B6 $(p<0.05)$ (data not shown).

B1-5 serotypes exhibited remarkable changes in positivity rates over time (Figure 2). The greatest variation was observed for B2 seroprevalence, which was the highest in 2004 (64.8\%, 95\% CI: 54.4-73.9) but significantly decreased in 2005 and 2006 (50.5\%, 95\% CI: 40.9-60.1, and 31.2\%, 95\% CI: 23.3-40.4), settling at low rates from 2007 to 2016 (range: 3.3\%-8.1\%) ( $p<0.0001)$. B1, B3, B4 and B5 instead showed a cyclical pattern, with periods of lower positivity rates followed by 1-3 years of increased seroprevalence. B3, B4 and B5 displayed a significant decrease in seroprevalence over years $(p<0.05)$. A specific trend was not evidenced for B6, due to its low seroprevalence never exceeding 5\%.

The fluctuating seroprevalence patterns over years seem to be partially reflected in nAb titers' distributions (Figure 2). NAb levels for B2 were higher in 2004-2006 but strongly decreased in the following years $(p<0.05)$, similarly to B2 seroprevalence. B3 titers were significantly higher in 2014 than most of the other years $(p<0.05)$, in correspondence of a seroprevalence peak. For B4 and B5 significantly lower levels were observed both for titers $(p<0.05)$ and seroprevalence in 2015. Differently, higher titer values were found for B5 in $2005(p<0.05)$, but without an increase in seroprevalence.

This article is protected by copyright. All rights reserved. 
Sex-related seroprevalence. Positivity rates stratified by sex reflected the general B1-6 seroprevalence, but the percentage of seropositivity was higher in females than in males (Figure 3a). This difference was significant for B2 and B3 $(p<0.05)$. The nAb levels were higher but not significant in females than in males (Figure 3b).

Age-related seroprevalence. Positivity for at least one serotype was lowest in toddlers aged 0-2 years (25.2\%; 95\% CI: 20.4-30.7), but strongly increased in preschool (3-5 years) (50.3\%; 95\% CI: 42.6-58.0) and school (6-10 years) children (70.4\%; 95\% CI: 63.1-76.8) ( $p<0.0001)$ (Figure 4). Seropositivity remained constantly high in the following groups, with the highest value observed at $41-50$ years (85.9\%; 95\% CI: 80.9-89.8), but decreased to $61.0 \%$ at $81-90$ years (95\% CI: $48.3-72.4)$. The number of individuals with multiple positivity also increased with age. In the 0-2 years group, nAb were directed against one serotype in 68.7\% (95\% CI: 56.8-78.5) of seropositive children, and against 2 and 3 serotypes respectively in 16.4\% (95\% CI: 9.4-27.1) and 7.5\% (95\% CI: 3.2-16.3) (Figure 4). At 3-5 years, 35.0\% (95\% CI: 25.545.9) of seropositive children had nAb for 2 viruses. The 41-50 years-old group showed the highest percentage of positivity for 3 viruses (22.4\%, 95\% CI: 17.2-28.6). Positivity to 4 and 5 viruses was observed in less than $6 \%$ of seropositive individuals, and positivity to all CVB was rare (less than $1.0 \%$ ).

The age-related positivity increase is significant also in analyzing B1-B5 specific seroprevalence. Interestingly, nAb titers distributions did not always reflect this trend (Figure 5). The 0-2 year group showed the lowest positivity rates, comprised between $10 \%$ and $12 \%$ for B3, B4 and B5. In the following age-groups, B4 had higher rates of seroprevalence compared to all other viruses, with a marked increase passing from preschoolers (25.2\%, 95\% CI: 19.1-32.4) to school children (40.2\%, 95\% CI: 33.147.8), and two peaks at 41-50 and 71-80 years. However, B4 nAb titers were higher in

This article is protected by copyright. All rights reserved. 
younger subjects, with a significant difference at 3-5 years with respect to age-groups over 10 years-old $(p<0.05)$, and lower at 71-80 years compared to groups under 60 years $(p<0.05)$. Only B4 maintained high seroprevalence in older ages. B3 and B5 showed a similar pattern with two peaks of increasing prevalence at 11-20 and 41-50 years, and a decrease in individuals older than 50 years. B3 nAb levels were significantly higher in children aged 3-5 years $(p<0.02)$ and lower starting from 61 years $(p<0.05)$. For B5 a significative difference was evidenced comparing $\mathrm{nAb}$ titers of the first 4 groups ( 0 -20 years) with the 51-60 years group ( $p<0.03)$, and titers of the first 2 groups (0-5 years) with the 61-70 and 71-80 year-old groups ( $p<0.05)$. B1 and B2 seroprevalence rates were always under $21 \%$ but peaked at different age-groups (6-10 and 41-50 years, respectively). No significant difference was observed in terms of nAb titers for these two serotypes. As far as B6 is concerned, a modest seroprevalence increase was observed at $41-50$ and $51-60$ years ( $4.2 \%$ and $4.6 \%)$ (data not shown).

\section{Discussion}

Clinical presentations of CVB are remarkably different and present various degrees of severity, from asymptomatic infection to meningitis and chronic inflammation of the heart and pancreas. No single serotype is linked to a specific disease. In addition, epidemiological and animal studies suggest that CVB could have a role in type 1 diabetes; to verify this hypothesis, the development of a human vaccine was recently announced $^{19}$.

In this context, the measurement of pre-existing immunity is important to deduce prevalence and to identify susceptible populations. Few recent studies focused on CVB seroprevalence in Europe ${ }^{20,21}$, and the last Italian work about this topic dates to 
$1991^{18}$. In this work a retrospective serologic study conducted in 2,459 individuals in Rome during a 13-year-long period is reported. Seroprevalence rates and nAb titers were analyzed in relation to gender and age of the subjects and to the year of observation, with the purpose of investigating the level of past exposure to the 6 CVB types.

Seropositivity for at least one virus was identified in $69.1 \%$ of samples, demonstrating the widespread diffusion of CVB infection in the study population. B4 was the most prevalent virus, with nearly half of the individuals having experienced the infection, followed by B3, B5, B1 and B2, while B6 nAb were detected sporadically. In 1991 in the area of Bari (South Italy), seroprevalence in children was higher for B1, B2 and B5 ${ }^{18}$. A European study conducted in 2001-2005 showed that B1-6 seroprevalence and ranking differed depending on the subjects' country, but B1 and B6 were the least represented CVB, similarly to our findings ${ }^{21}$. These differences are not surprising, since Enterovirus diffusion is characterized by temporal and geographical variation, preventing the confrontation of data between different years and location ${ }^{22}$. As a confirmation of this, the positivity values were not steady, but rather changed over years. In fact, B1, B3, B4 and B5 seroprevalence patterns underwent cyclical variations that could reflect differences in circulation, consistently with CVB epidemiology and with several surveillance works ${ }^{12-15,23,24}$. The most noticeable difference was observed for B2 seroprevalence, which decreased from 65\% in 2004 to levels lower than 8.5\% starting from 2007, being replaced by B4 as seroprevalent type. To our knowledge, a decline in B2 seroprevalence in 2004-2007 is not documented elsewhere. This trend could reflect a period of wider circulation during and/or prior to 2004, followed by a decrease in the number of infections. An increase in B2 detection was observed in a surveillance study in 2004 in France ${ }^{23}$, and B2 had 
the highest seroprevalence in individuals from Sweden and Greece in 2001-2005 ${ }^{21}$. Unfortunately, recent data about B2 surveillance or prevalence in Italy are not available. The median age of patients was higher in 2004 (data not shown), so the higher seroprevalence observed for B2 in that year could be attributed to a different age distribution of population. Moreover, it cannot be excluded that the decrease in B2 nAb detection was due to the circulation of a "new" strain with different antigenic properties and able to elicit a different nAb response.

The 6 serotypes determined nAb production with different intensities. B3 and B4 stimulated the strongest responses, while nAb levels were lower for B1 and B6. Similar results were reported by Oikairinen et al. ${ }^{21}$. In another work analyzing B3 and $\mathrm{B} 5$ prevalence, $\mathrm{nAb}$ GMT of B3 was higher than $\mathrm{B} 5^{25}$. Repeated immune stimulations due to the wide diffusion of B3 and B4 could contribute to maintaining high levels of $\mathrm{nAb}$ in subjects who previously experienced the infection. On the contrary, a low production of B1 and B6 nAb could reflect their lower diffusion or their ability to escape the immune response.

Host factors also influence nAb levels. In this study higher values were detected in females, and similar data were obtained for B4-IgG in children ${ }^{26}$. Females had also higher seroprevalence values, and the difference was significant for B2 and B3. However, other works did not report significant gender-specific differences in CVB positivity ${ }^{18,25,27}$. Increasing number of evidences suggest that female subjects produce a more intense immune response during viral infections, maintaining it also after the pathogen's clearance ${ }^{28}$. Mechanisms involved in sex-related difference in immunity are mostly unknown, but our results probably reflect this phenomenon.

This article is protected by copyright. All rights reserved. 
The study clearly evidences that positivity for at least one serotype increases with age, together with the number of individuals having experienced infection with more than one virus. These findings are consistent with the observations of other groups $^{18,21,25,27,29}$. Preschoolers are the subjects at higher risk of infection: about $75 \%$ of children aged 0-2 years and 50\% of children aged 3-5 years had never experienced CVB infection. Since no vaccine is available for CVB, nurseries and schools probably have an important role in the transmission of infection in children. In addition, a higher nAb production for B3 and B4 was observed in children aged 3-5 years. The same observation was reported for B3 in a Chinese population ${ }^{25}$. A stronger antibody response is probably stimulated by the high viral circulation in this age-group or reflects a recent primary infection. On the contrary, B4, B3 and B5 nAb levels were lower in older age-groups, compatibly with a condition of reduced adaptive immune function in these subjects ${ }^{30}$.

In conclusion, the great number of samples included in the analysis allowed the gathering of comprehensive information on CVB epidemiology, highlighting serospecific seroprevalence variations over time and in different age-groups. Serologic data are valuable to assess the risk of outbreaks and for the development of future immunization programs. Limitations of this work are the lack of clinical information for the whole population and the fact that the study cannot be considered as a serosurvey of healthy subjects. Moreover, the analysis is not based on a multi-center experience, therefore our data could not represent the seroprevalence in the Italian population. However, considering that the results derived from one of the 3 largest public and academic hospital in Italy, and the lack of studies on CVB seroprevalence in Italy, this work provides a well characterized seroepidemiologic insight and underlines the need for a continuous monitoring of CVB epidemiology. 


\section{Aknowledgments}

Funding: none

Conflict of Interest: the authors declare that they have no conflict of interest.

\section{Author's contribution}

GA and OT conceived the work. SI and FF wrote the text. PM, GT, DDC, LM and TM performed the assays. GG, GA and OT critically revised the manuscript. All authors contributed to manuscript revision, read and approved the final version.

\section{References}

1. Esposito S, Principi N. Hand, foot and mouth disease: current knowledge on clinical manifestations, epidemiology, aetiology and prevention. Eur J Clin Microbiol Infect Dis 2018; 37:391-8.

2. Tao Z, Wang H, Li Y, Liu G, Xu A, Lin X, Song L, Ji F, Wang S, Cui N, Song Y. Molecular epidemiology of human enterovirus associated with aseptic meningitis in Shandong Province, China, 2006-2012. PloS One 2014; 9:e89766.

3. Angez M, Shaukat S, Zahra R, Alam MM, Sharif S, Khurshid A, Arshad Y, Suleman M, Mujtaba G, Zaidi SSZ. Characterization of group B coxsackieviruses isolated from non-polio acute flaccid paralysis patients in Pakistan: vital assessment before polio eradication. Epidemiol Infect 2017; 145:2473-2481.

4. Huber S, Ramsingh AI. Coxsackievirus-induced pancreatitis. Viral Immunol 2004; $17: 358-369$

5. Romero JR. Pediatric group B coxsackievirus infections. Curr Topics Microbiol Immunol 2008; 323:223-239.

This article is protected by copyright. All rights reserved. 
6. Sin J, Mangale V, Thienphrapa W, Gottlieb RA, Feuer R. Recent progress in understanding coxsackievirus replication, dissemination, and pathogenesis. Virology 2015; 484:288-304.

7. Flynn CT, Kimura T, Frimpong-Boateng K, Harkins S, Whitton JL. Immunological and pathological consequences of coxsackievirus RNA persistence in the heart. Virology 2017; 512:104-112.

8. Alidjinou EK, Engelmann I, Bossu J, Villenet C, Figeac M, Romond MB, Sanè F, Hober D. Persistence of Coxsackievirus B4 in pancreatic ductal-like cells results in cellular and viral changes. Virulence 2017; 8:1229-1244.

9. Hyöty H, Leon F, Knip M. Developing a vaccine for type 1 diabetes by targeting coxsackievirus B. Expert Rev Vaccines 2018; 17:1071-1083.

10. Rodriguez-Calvo T. Enterovirus infection and type 1 diabetes: unraveling the crime scene. Clin Exp Immunol 2019; 19:15-24.

11. Stone VM, Hankaniemi MM, Svedin E, Sioofy-Khojine A, Oikarinen S, Hyöty H, Laitinen OH, Hytönen VP, Flodström-Tullberg M. A Coxsackievirus B vaccine protects against virus-induced diabetes in an experimental mouse model of type 1 diabetes. Diabetologia 2018; 61:476-481.

12. Khetsuriani N, LaMonte-Fowlkes A, Oberst S, Pallansch MA, Centers for Disease Control and Prevention. Enterovirus surveillance-United States, 1970-2005. MMWR Surveill Summ 2006; 55:1-20.

13. Centers for Disease Control and Prevention. Nonpolio enterovirus and human parechovirus surveillance-United States, 2006-2008. MMWR Morb Mortal Wkly Rep 2010; 59:1577.

14. Abedi GR, Watson JT, Pham H, Nix WA, Oberste MS, Gerber SI. Enterovirus and human parechovirus surveillance-United States, 2009-2013. MMWR Morb Mortal Wkly Rep 2015; 64:940-3.

This article is protected by copyright. All rights reserved. 
15. Abedi GR, Watson JT, Nix WA, Oberste MS, Gerber SI. Enterovirus and parechovirus surveillance-United States, 2014-2016. MMWR Morb Mortal Wkly Rep 2018; 67:515.

16. Lugo D, Krogstad P. Enteroviruses in the early 21st century: new manifestations and challenges. Curr Opin Pediatr 2016; 28:107.

17. Kim H, Kang B, Hwang S, Hong J, Chung J, Kim S, Jeong YS, Kim K, Cheon DS. Molecular characteristics of human coxsackievirus B1 infection in Korea, 2008-2009. J Med Virol 2013; 85:110-5.

18. Leogrande G. Studies on the epidemiology of child infections in the Bari area. Current state of immunity against coxsackie B viruses. Int J Clin Lab Res 1991; 21:95-103.

19. Dunne JL, Richardson SJ, Atkinson MA, Craig ME, Dahl-Jørgensen K, Flodström-Tullberg M, Hyöty H, Insel RA, Lernmark A, Lloyd RE, Morgan NG, Pugliese A. Rationale for enteroviral vaccination and antiviral therapies in human type 1 diabetes. Diabetologia 2019; 62:744-753.

20. Mavrouli MD, Spanakis N, Levidiotou S, Politi C, Alexiou S, Tseliou P, Hatzitaki M, Foundouli K, Tsakris A, Legakis NJ, Routsias JG. Serologic prevalence of coxsackievirus group B in Greece. Viral Immunol 2007; 20:11-8.

21. Oikarinen S, Tauriainen S, Hober D, Lucas B, Vazeou A, Sioofy-Khojine A, Bozas E, Muir P, Honkanen H, Ilonen J, Knip M, Keskinen P, Saha MT, Huhtala H, Stanway G, Bartsocas C, Ludvigsson J, Taylor K, Hyöty H, the VirDiab Study Group. Virus antibody survey in different European populations indicates risk association between coxsackievirus B1 and type 1 diabetes. Diabetes 2014; 63:655-662.

22. Palacios G, Oberste MS. Enteroviruses as agents of emerging infectious diseases. J Neurovirol 2005; 11:424-433.

This article is protected by copyright. All rights reserved. 
23. Antona D, Lévêque N, Chomel JJ, Dubrou S, Lévy-Bruhl D, Lina B. Surveillance of enteroviruses in France, 2000-2004. Eur J Clin Microbiol Infect Dis 2007; 26:403-412.

24. van der Sanden SMG, Koopmans MPG, van der Avoort HGAM. Detection of human enteroviruses and parechoviruses as part of the national enterovirus surveillance in the Netherlands, 1996-2011. Eur J Clin Microbiol Infect Dis 2013; 32:1525-1531.

25. Zhu R, Cheng T, Yin Z, Liu D, Xu L, Li Y, Wang W, Liu J, Que Y, Ye X, Tang Q, Zhao Q, Ge S, He S, Xia N. Serological survey of neutralizing antibodies to eight major enteroviruses among healthy population. Emerg Microbes Infect 2018; $7: 1-15$

26. Viskari H, Ludvigsson J, Uibo R, Salur L, Marciulionyte D, Hermann R, Soltesz G, Füchtenbusch M, Ziegler AG, Kondrashova A, Romanov A, Knip M, Hyöty H. Relationship between the incidence of type 1 diabetes and enterovirus infections in different European populations: results from the EPIVIR project. J Med Virol 2004; 72:610-7.

27. Tao Z, Li B, Xu A, Liu Y, Song L, Wang S, Xiong $\mathrm{P}$, Lin X, Song Y. Seroprevalence of coxsackievirus B3 in Yantai, China. Jpn J Infect Dis 2013; $66: 537-8$.

28. Markle JG, Fish EN. SeXX matters in immunity. Trends Immunol 2014; 35:97104.

29. Sun Y, Miao Z, Yan J, Gong L, Chen Y, Chen Y, Mao HY, Zhang YJ. Seromolecular epidemiology of enterovirus-associated encephalitis in Zhejiang Province, China, from 2014 to 2017. Int J Infect Dis 2019; 79:58-64.

This article is protected by copyright. All rights reserved. 
30. Pera A, Campos C, López N, Hassouneh F, Alonso C, Tarazona R, Solana F. Immunosenescence: implications for response to infection and vaccination in older people. Maturitas 2015; 82:50-5.

\section{Figure legends}

Fig. 1 CVB seroprevalence from 2004 to 2016

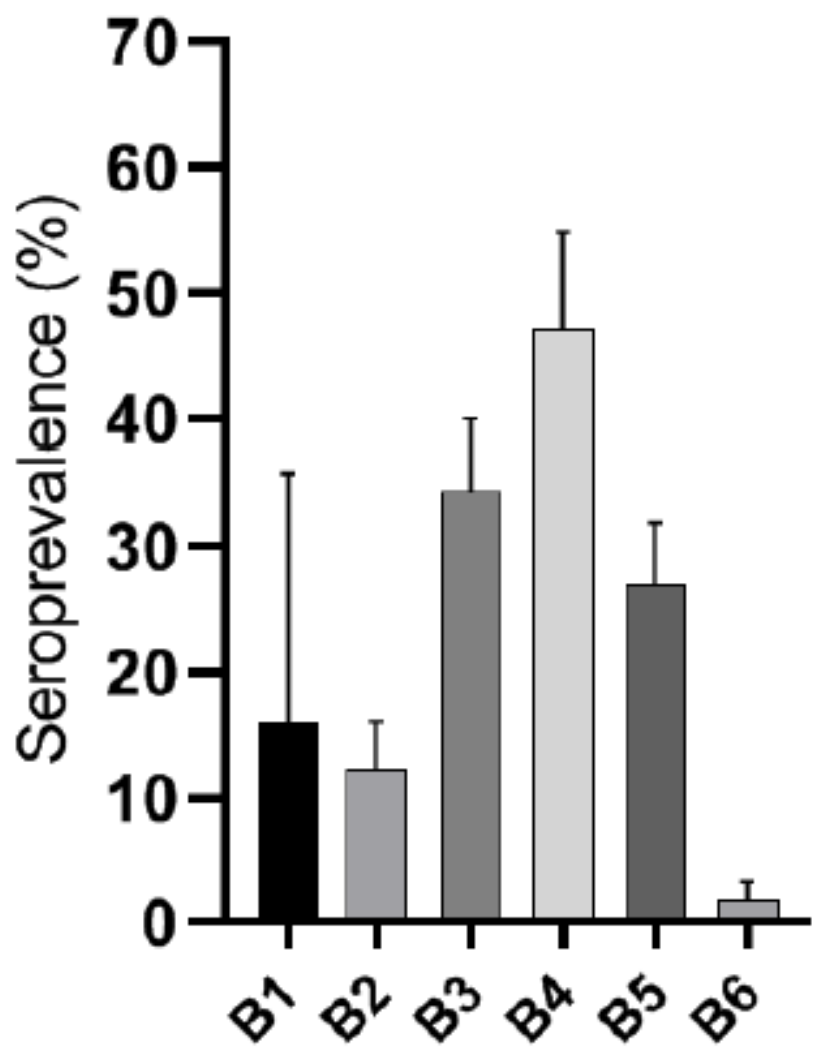

This article is protected by copyright. All rights reserved. 
Fig. 2 B1-6 seroprevalence trends (dashed lines) and nAb titers' distributions (box plots) for each year of observation (2004/2016). Box plots show the minimum, first quartile, median, third quartile and maximum logarithmic titer levels
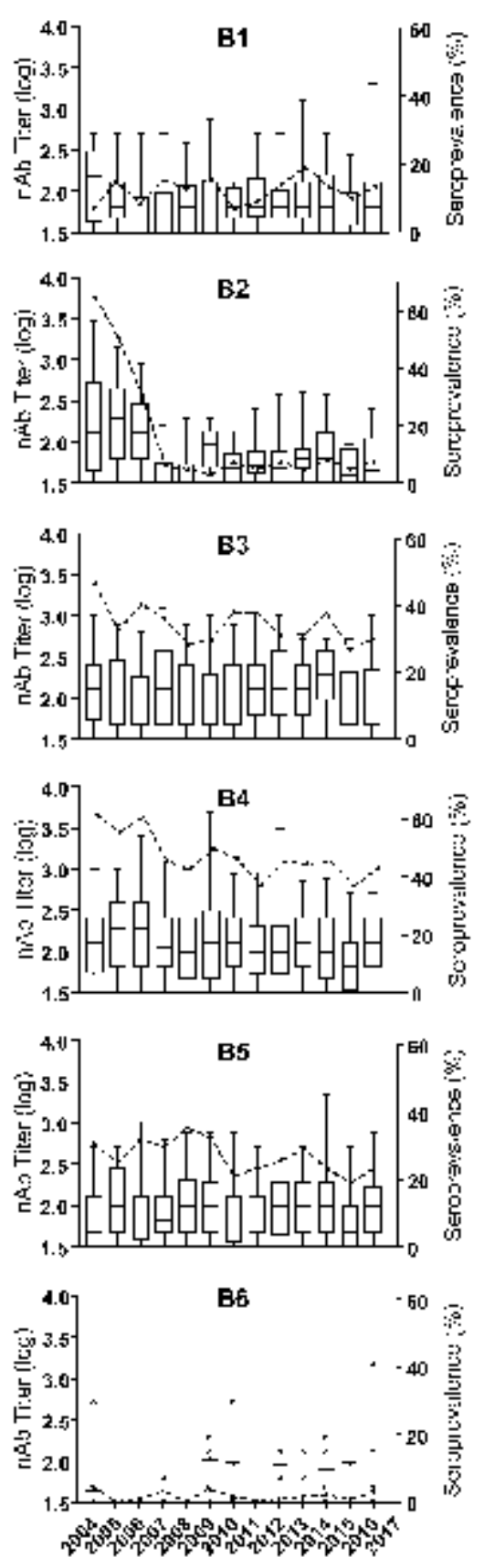

Year

This article is protected by copyright. All rights reserved. 
Fig. 3 NAb titers' distributions (a) and seroprevalence (b) of CVB in male and female subjects $\left({ }^{*} p<0.05\right)$. The box plots show the minimum, first quartile, median, third quartile and maximum logarithmic titer levels
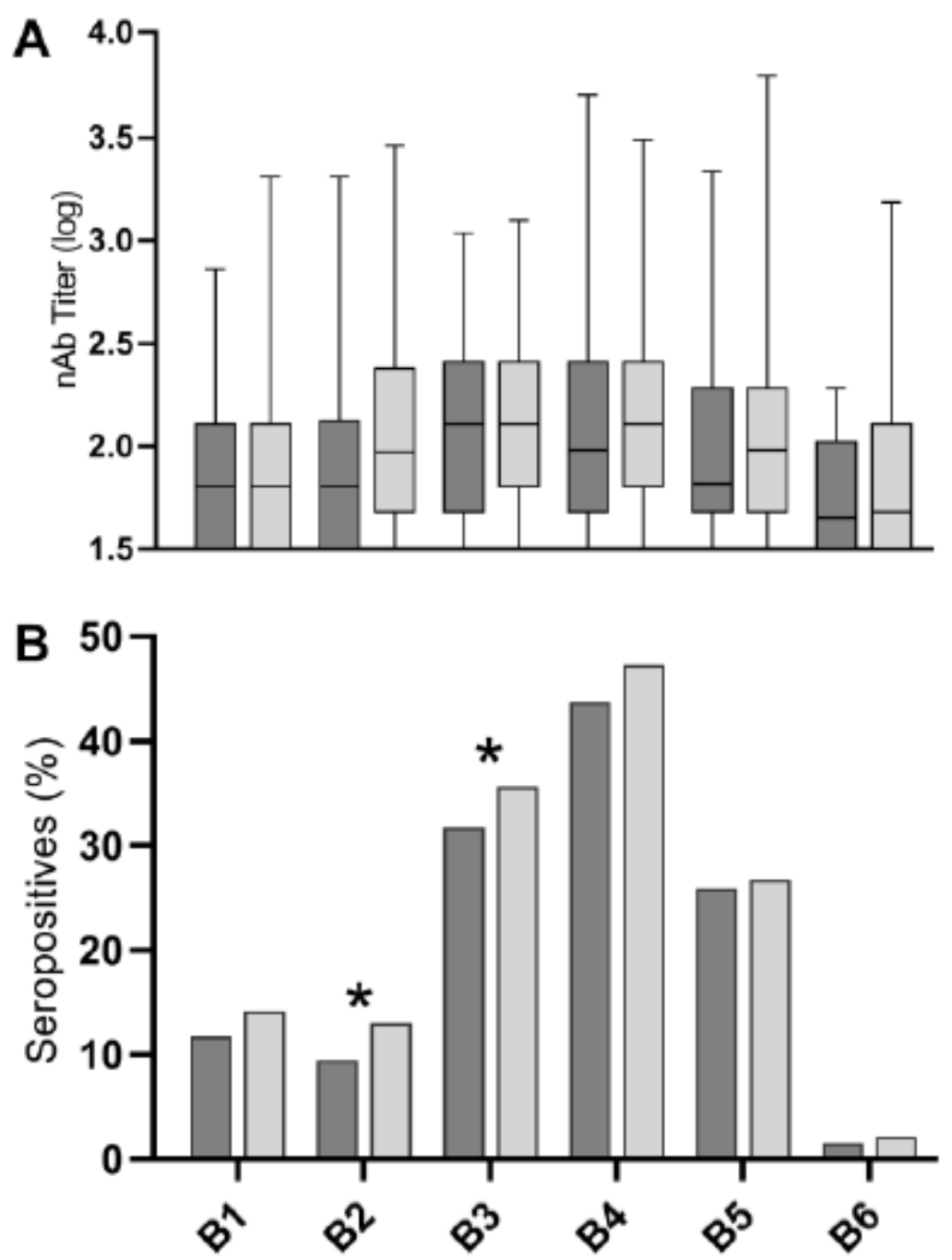

This article is protected by copyright. All rights reserved. 
Fig. 4 Proportions of individuals seropositive to one or more CVB classified for agegroup

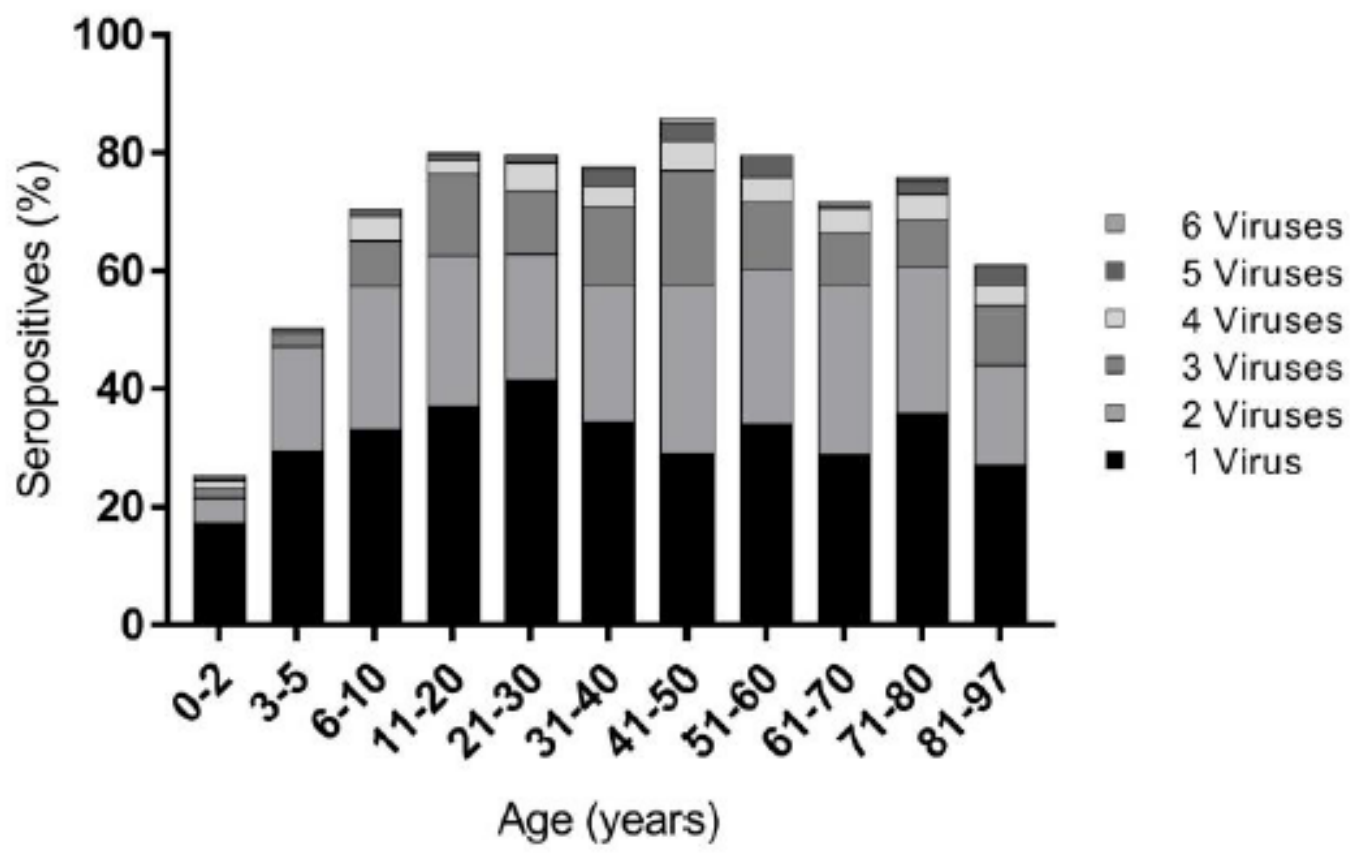

This article is protected by copyright. All rights reserved. 
Fig. 5 B1-5 seroprevalence trends (dashed lines) and nAb titers' distributions (box plots) observed in age-groups. Box plots show the minimum, first quartile, median, third quartile and maximum logarithmic titer levels
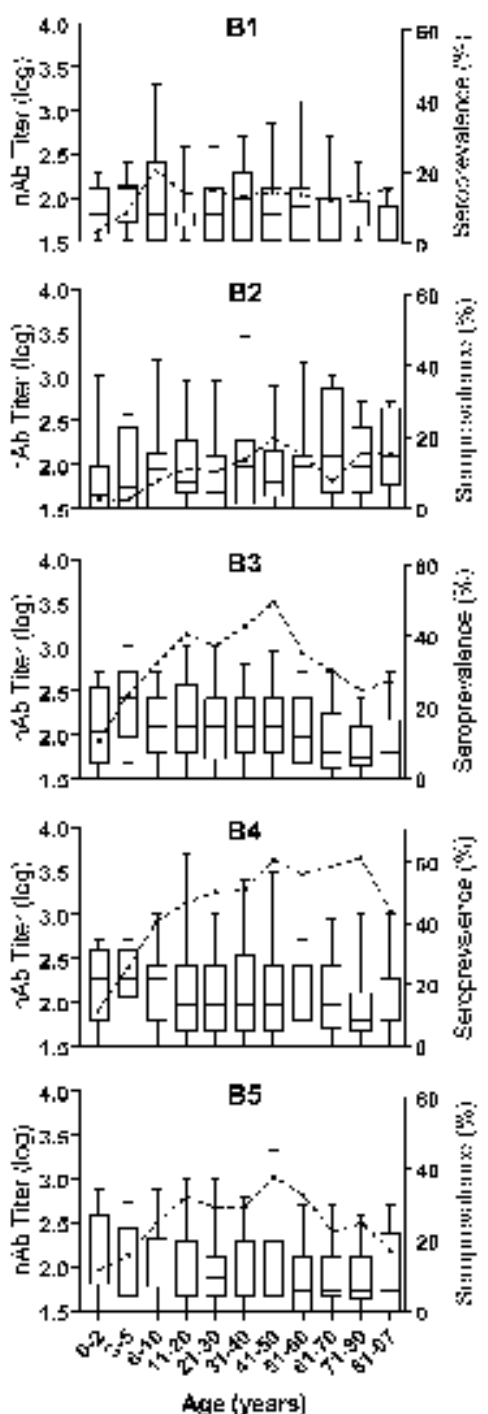

This article is protected by copyright. All rights reserved. 